\title{
Contents of Unconscious Color Perception
}

\author{
Błażej Skrzypulec ${ }^{1}$ (1)
}

Accepted: 12 April 2021 /Published online: 27 April 2021

(C) The Author(s) 2021

\begin{abstract}
In the contemporary discussions concerning unconscious perception it is not uncommon to postulate that content and phenomenal character are 'orthogonal', i.e., there is no type of content which is essentially conscious, but instead, every representational content can be either conscious or not. Furthermore, this is not merely treated as a thesis justified by theoretical investigations, but as supported by empirical considerations concerning the actual functioning of the human cognition. In this paper, I address unconscious color perception and argue for a negative thesis - that the main experimental paradigms used in studying unconscious color perception do not provide support for the position that conscious and unconscious color representations have the same type of content. More specifically, I claim that there is no significant support for the claim that unconscious vision categorically represents surface colors.
\end{abstract}

Keywords Unconscious perception - Color perception · Blindsight · Representational content $\cdot$ Metacontrast masking $\cdot$ Higher-order theories of consciousness

As a result of severe damage to the primary visual cortex, a person may report not having any conscious visual experiences in a certain part of the visual field. It seems that when asked about visual properties of objects positioned in an affected fragment of the visual field, such a person can only guess. However, when confronted with a forced-choice task in which she must choose one of two options, for instance whether the presented object is green or red, the 'guessing' is significantly accurate (e.g., Weiskrantz 2009). This phenomenon, known as 'blindsight', is one of the empirical phenomena that suggest the presence of unconscious perception of color. Nevertheless, the thesis of the existence of unconscious color perception is hardly without controversies. First, controlling whether participants have conscious experience of a presented stimulus is methodologically challenging, and so it may be doubted if unconscious color perception is really unconscious (e.g., Ramsøy and Overgaard 2004; Reingold

Błażej Skrzypulec

blazej.skrzypulec@uj.edu.pl; https://orcid.org/0000-0003-2780-5477

1 Institute of Philosophy, Jagiellonian University, Kraków, Poland 
2004; Snodgrass 2004). For instance, participants may be prone to provide conservative responses that they do not see anything even though some weak form of consciousness is in fact present (Phillips 2016). Second, even if one accepts that phenomena such as blindsight involve unconscious processing of sensory information, it may still be questioned whether they are perceptual phenomena (see Phillips 2018; Taylor 2019). For example, one may claim that blindsight is a subpersonal phenomenon which, unlike genuine perceptual experiences, is not a state of an organism but of one of its parts (see Phillips 2020; Quilty-Dunn 2019 for discussion). However, there is also a potential third doubt concerning unconscious color perception, which has attracted less attention. It may be proposed that even if phenomena such as blindsight are perceptual and unconscious, they should not be classified as a perception of colors, or at least not as a perception of colors understood in the same way as in usual conscious vision.

Within the representationalist framework, this last question can be understood as concerning the contents of unconscious color perception, namely, whether contents of unconscious color representations are of the same type as contents of conscious color representations. In this paper, I argue for a negative thesis - that the main experimental paradigms used in studying unconscious color perception do not provide support for the position that conscious and unconscious color representations have the same type of content. More specifically, I distinguish between (a) representing surface colors and reflected colors, and (b) representing colors relationally and categorically. According to the first distinction (e.g., Kentridge 2015; Norman et al. 2014), vision may represent surface colors, i.e. properties that remain the same despite changes in scene illumination. For instance, due to the function of mechanisms responsible for color constancy, a property may be represented as a white color both when a scene is highly illuminated and when the considered surface is shaded. On the other hand, when such constancy mechanisms are not available, only a reflected color can be represented, whose characteristics are wholly determined by parameters of light reaching eyes without separating the illumination from the reflectance properties of a surface. According to the second distinction, colors can be represented relationally or categorically. A relational representation represents a color as standing in certain relations to other colors. For instance, it may be represented that a color $X$ is highly similar to a color $Y$. However, colors can also be represented categorically, such that a color is represented as a token of a certain color-type. For example, it may be represented that color $X$ belongs to type 'red' while color $Y$ belongs to type 'orange'.

It is plausible to assume that conscious vision can represent surface colors and to do so both relationally and categorically. I argue that, on the contrary, there is no strong evidence for an analogous claim concerning unconscious color perception. In consequence, according to the current state of knowledge, it is possible that the contents of unconscious color representations are of a different type than contents of conscious color representations. To establish my claim, I discuss four research paradigms which play major roles in philosophical consideration of unconscious color perception: (a) forced-choice studies on blindsight (e.g., Boyer et al. 2005; Kentridge et al. 2007), (b) metacontrast masking studies (e.g., Norman et al. 2014; Ro et al. 2009), (c) priming studies on visual neglect (e.g., Cohen et al. 1995; Kristjánsson et al. 2009), and (d) studies concerning dichoptic color fusion (e.g., Fogelson et al. 2014; Moutoussis and Zeki 2002). 
The proposed negative thesis is not without relevance for contemporary discussions on the philosophy of mind, as there are influential theories postulating that conscious and unconscious perception do not differ in type of content. In particular, it is proposed that content and phenomenal character are 'orthogonal', i.e. there is no type of content which is essentially conscious, but instead, every type of representational content can be either conscious or not (e.g., Schlicht 2012; Vosgerau et al. 2008). The thesis that conscious and unconscious experiences have the same type of content is a common assumption made in higher-order theories of consciousness, according to which a state is conscious in virtue of being represented by a higher-order state (see Peebles 2017 for a review of major theories of phenomenal consciousness). On the grounds of higherorder theories it seems natural to postulate that conscious and unconscious perceptual states have the same type of content, as they differ only in respect of the fact that the conscious state is also additionally represented by a higher-order mental state. Furthermore, it is not uncommon for proponents of the orthogonality between phenomenology and content to suggest that their position is supported by the empirical results obtained in investigations concerning unconscious perception (e.g., Rosenthal 2005, pp. 108109, 187). Nevertheless, I argue that this is not the case, at least when color perception is considered.

I start (Section 1) by explicating the differences between types of color representation contents and introducing (Section 2) the equivalence thesis, which states that conscious and unconscious color perception have the same type of content. Subsequently, in Sections 3, 4 and 5, I discuss blindsight studies, metacontrast masking studies, neglect studies, and dichoptic color fusion studies to demonstrate that they do not provide support for the equivalence thesis.

\section{Types of Color Contents}

Color space is a space in which every point is a single color, distinct points are distinct colors, and there are no colors that are not located within this space. Colors within a color space are organized such that the distance between colors corresponds to their perceptual similarity (e.g., a shade of red is closer to a shade of orange than to a shade of green). According to usual characterizations (see Palmer 1999, pp. 97-99), a color space is three-dimensional, as every color is specified by its hue, saturation, and lightness. For instance, two shades of red may have the same hue but be distinct colors as they differ in saturation or lightness. Similarly, a shade of green is a different color from a shade of red even if their lightness and saturation match, as they differ in hue.

In virtue of its structure, color space contains relational information about colors. Such relational information concerns how colors are related to each other-for instance if a color $X$ is closer within the color space to color $Y$ than to color $Z$, then $X$ is more perceptually similar to $Y$ than to $Z$. Analogously, if a color $X$ occupies the same place in the color space as a color $Y$, then $X$ is identical to $Y$. Furthermore, the color space contains also categorical information about colors which concern a category, such as 'red' or 'green', to which a color belongs. It is so because a category to which a color belongs is determined by its position in color space, as the color space can be divided into topologically connected, disjoint parts which correspond to various color categories. For instance, there is a part of the color space such that colors positioned within it belong 
to the category 'red' and another, nonoverlapping part such that colors located in it belong to the category 'green'.

The distinction between relational and categorical information allow one to distinguish two types of color representations. Colors can be represented relationally by representing their relations to other colors; for instance, that a color $X$ is highly similar to a color $Y$. However, colors can also be represented categorically by representing to which color category they belong, for example that a color $X$ is a shade of red.

These types of color representations are independent in the sense that color can be represented relationally while not being represented categorically. For instance, a color $X$ may be represented as having a high level of similarity to a color $Y$ without being represented as belonging to the category 'red'. It should be noted that the above independence does not rely on the possibility of an inverted spectrum, i.e. a situation in which color categories are modified, for instance the category 'red' is swapped with the category 'green', without any modification in relations between colors within the color space. Even if an inverted spectrum is impossible because a pattern of relation in which a color $X$ stands to other colors in the color space fully determines a category to which $X$ belongs, it is still possible that $X$ is represented relationally but not categorically. It is so because representing introduces an intensional context. For instance, using the classic philosophical example, while it is true that Clark Kent is identical to Superman, one can mentally represent that a given man is Clark Kent without representing that this man is Superman. Analogously, even if for a color $X$ standing in certain relations entails belonging to the 'red' category, it may be represented that $X$ stands in certain relations without representing to which category it belongs.

Similarly, differentiating between colors, by representing color $X$ as not identical to color $Y$, does not necessarily require categorical color perception, because to represent the nonidentity of $X$ and $Y$ it is sufficient to recognize that they occupy distinct places within the color space. Succeeding in such differentiation task does not require an additional recognition that colors $X$ and $Y$ lay in fragments of the color space corresponding to some color categories. In fact, color differentiation can occur even if the representing cognitive system does not divide color space into any fragments corresponding to color categories. Of course, that is not to deny, as suggested by the literature on color naming (e.g., Clifford et al. 2012; Davidoff and Fagot 2010; Kay and Regier 2006), that the various ways of dividing color space into categories may influence how the similarity of colors is perceived and how colors are discerned from each other. However, the presence of such influences does not undermine the general thesis that representing identity - or nonidentity - between colors is possible without representing colors as members of some color categories. Due to the above independence, it is possible that there are color representations with different types of content. In particular, there may be representations which represent colors only in a relational way (e.g., $X$ is similar to $Y$ ) and such that represent colors both relationally and categorically (e.g., $X$ is similar to $Y$ and $X$ is a shade of red). The latter option is intuitively true of typical cases of conscious color perception.

Categorical color perception is often discussed in the context of linguistic influence on how we perceive colors, as the presence of such influences may support a Whorfian thesis that language determines how we experience the world. For instance, it has been observed that the time needed to notice a difference between pairs of colors is shorter for pairs that cross a boundary between linguistically determined categories, even if the distance in 
color space between elements of a pair is equal (see Ocelák 2016 for a review). However, dividing colors into color categories is not merely a linguistic but also a perceptual phenomenon. There are two types of result which suggest that perception represents colors as belonging to distinct categories independent of linguistic classifications. First, studies of infants show categorical effects in color perception that occur before language acquisition and are largely independent from cultural context (Franklin et al. 2005a, b, 2009). For example, it is well-recognized that infants look longer at a novel stimulus but the novelty effect disappears when an infant becomes habituated to a stimulus. It has been shown that when infant is habituated to a color, then habituation also occurs for colors from the same category, but not for colors from different categories, even if the distances in color space are equal (Franklin and Davies 2004). The second type of evidence comes from electrophysiological studies concerning an event-related potential known as visual mismatch negativity, which indicates pre-attentive visual change detection (e.g., Clifford et al. 2010; Thierry et al. 2009). When people are presented with a change detection task in which a change consists of replacing a color with a color from the same category or with a color from a different category, a greater visual mismatch negativity is recorded when a different-category change occurs. Because visual mismatch negativity indicates early, preattentive change detection, such results suggest that categorical differences are processed before top-down, linguistic influences can occur.

Apart from the distinction between relational and categorical representation, there is also a second distinction which differentiates types of contents of color vision. In order to represent colors, vision must process information provided by the light reaching retinas. This information is determined by two primary sources: the parameters of illumination and the parameters of surfaces from which light is reflected. In usual cases of conscious perception, the visual system attempts to separate the influence of illumination from the influence of reflectance properties of a surface and represents a 'surface color', i.e. a reflectance property which remains the same despite changes in illumination. This is achieved by mechanisms of color constancy using various heuristics which, for instance, allow distinguishing illumination edges from reflectance edges, utilize assumptions about typical properties of natural illumination, or compare relative differences between neighboring surfaces (see Palmer 1999, pp. 125-136). On the other hand, if constancy mechanisms were unavailable, only 'reflected colors', which are determined by light coming from a surface without separation of illumination and reflectance components, could be represented. In other words, a system with functioning constancy mechanisms aims to represent a colorful stimulus as having a property which remains the same despite changes in illumination. However, a system without constancy mechanisms cannot represent a property of a colorful stimulus in this way and so color representations of such a system do not have the same type of content as a system with abilities for constancy perception. In subsequent sections, I assume that, in typical cases, conscious vision categorically represents surface colors, and I investigate whether there is support for an analogous claim that unconscious color vision is also able to represent surface colors categorically.

\section{Equivalence Thesis}

If empirical investigations do not provide support for unconscious categorical representations of surface colors, this lack is problematic for a proponent of the 'equivalence 
thesis', stating that conscious and unconscious color representations have the same type of content. In this case one can, without being in conflict with scientific knowledge, claim that the current empirical evidence is insufficient to justify the equivalence thesis. In other words, a proponent of the equivalence thesis cannot refer to empirical results in order to show that her position has stronger scientific foundation than the alternative accounts.

Below, I treat the equivalence thesis as a statement concerning the qualitative differences between types of content and not quantitative differences regarding performance of conscious and unconscious vision. For instance, it may be the case that both conscious and unconscious vision represent colors categorically but conscious vision may simultaneously represent more colorful objects than unconscious vision, or that conscious categorical color perception is more fine-grained in terms of the number of categories to which colors are assigned. Further, I do not treat such quantitative differences as posing a problem for the equivalence thesis regarding types of content. It should be noted that my reasoning does not assume that all conscious color representations categorically present surface colors. The equivalence thesis states that for each conscious representation there is an unconscious representation with content of the same type, so there is no type of content that can be solely conscious. In consequence, if there are at least some conscious color representations presenting surface colors in the categorical way, but there is no empirical support that there is unconscious color vision with contents of the same type, then my negative position is justified.

The equivalence thesis is often formulated in the context of higher-order theories of consciousness according to which a mental state $X$ is conscious in virtue of being represented by a higher-order state $Y$. For instance, Rosenthal, one of the main proponents of the higher-order theories, expresses the equivalence thesis in terms of sensory qualities (Rosenthal 2005, pp. 142-143). Sensory qualities are properties of mental states which stand in a systematic relationship to physical properties of objects. More specifically, there are distinct types of sensory qualities, like color, which correspond to types of physical properties, like surface properties related to light reflectance. For instance, a red sensory quality corresponds to a certain surface property, a green sensory quality corresponds to a distinct surface property, and a distance between them in the perceptual color space corresponds to the difference between the way in which the considered surface properties reflect light. In representationalist terms, which are not explicitly adopted by Rosenthal, it can be stated that sensory qualities represent some physical properties of perceived objects.

According to Rosenthal, being conscious is not an essential characteristic of sensory qualities. In fact, he claims that being a sensory quality and being conscious are independent features because a state is not conscious due to its specific sensory qualities but due to being represented by a higher-order state (Rosenthal 2005, p. 142). In consequence, for each conscious state with a sensory quality $S$ there is also a possible unconscious state with the same sensory quality. If unconscious perceptual states represent colors only relationally or represent only reflected colors, then such an equivalence thesis is false when applied to the actual capabilities of the human perception: there are certain sensory qualities, in particular representing division of colors into categories or representing sameness of colors despite illumination changes, which are possessed only by some of the conscious states. 
The higher-order theories of consciousness are not the only ones which are interpreted as involving the equivalence thesis. For instance, Berger and Nanay (2016) suggest the equivalence thesis in the context of Tye's (1995) PANIC theory, in which conscious content is such that is abstract, nonconceptual, intentional, and is appropriately poised to impact cognition and behavior. Within the framework of PANIC theory it seems plausible to assume that no content is essentially conscious, as the same content can be appropriately poised (and so be conscious) or not be poised in such a way (and so be unconscious). The equivalence thesis is also formulated independently from any particular theory of consciousness in terms of orthogonality of content and consciousness (see Schlicht 2012; Vosgerau et al. 2008). This orthogonality means that every content can be combined both with being conscious and being unconscious and so there is no type of content that must be conscious.

From the perspective of this paper, it is especially interesting that the equivalence thesis is not treated merely as a thesis justified by theoretical investigations, but as supported by empirical considerations concerning actual functioning of the human cognition. For instance, it is believed that a thesis that the same sensory qualities can be both conscious and unconscious is supported by phenomena such as blindsight and the results of priming studies (Rosenthal 2005, pp. 108-109, 187; Rosenthal 2010; Vosgerau et al. 2008). More generally, in philosophical works concerning unconscious perception, four types of empirical investigations are commonly acknowledged as being the most important in justifying the presence of unconscious color perception: (a) studies on blindsight involving a forced-choice task (Berger and Nanay 2016; Brogaard 2011; Dretske 2006; Phillips 2016; Zięba 2019), (b) metacontrast masking studies (Anaya and Clarke 2017; Phillips 2018; Rosenthal 2010; Taylor 2019), (c) studies concerning visual neglect (Phillips 2016), and (d) investigations demonstrating dichoptic color fusion (Block 2015). In the following sections, I discuss these types of studies in order to show that they do not provide a significant support for the equivalence thesis.

\section{Blindsight and Surface Colors}

Blindsight is a phenomenon which occurs when a person, due to severe disturbances in the function of the primary visual cortex, reports having no phenomenal consciousness in a fragment of the visual field but is nonetheless able to succeed in a forced-choice task when a stimulus is presented in the blind fragment. ${ }^{1}$ In such a task, the participant knows what stimuli can be presented (usually two options, like red color and green color are available), and in each trial, the participant is asked to decide which stimulus was actually presented even if uncertain of the correct answer. While blindsighted individuals subjectively resort to guessing, their responses are not distributed randomly, with accurate responses significantly more common than incorrect responses (see Cowey 2004 for a review). Traditionally, the blindsight phenomenon has been investigated in patients with a damaged primary visual cortex, but blindsight can also be induced temporally by transcranial magnetic stimulation (e.g., Boyer et al. 2005). It is

\footnotetext{
${ }^{1}$ More precisely, two types of blindsight are distinguished. In type 1 blindsight, a person does not report any awareness of stimuli, while in type 2 blindsight, a person reports some sort of feeling when the visual stimulus changes but without the form of a visual experience (e.g., Kentridge 2015).
} 
well-established that individuals with blindsight can succeed in forced-choice tasks when presented with various types of visual stimuli including colorful stimuli (e.g., Brent et al. 1994; Stoering and Cowey 1992; Weiskrantz 2009). Thus, the occurrence of blindsight is often treated as evidence for the presence of unconscious color perception.

According to the current state of knowledge, it is clear that color vision in blindsight is not simply normal color vision without consciousness (see Weiskrantz 2009). For instance, the threshold for detecting wavelength differences is higher in blindsight (Stoering and Cowey 1992) and the number of color categories that can be distinguished is lower than in conscious vision (Brent et al. 1994). Nevertheless, such differences can be easily interpreted as quantitative differences concerning performance of conscious and unconscious vision and do not pose a problem for the qualitative equivalence thesis. Furthermore, success in forced-choice tasks studies suggests that individuals with blindsight retain some form of categorical color perception. If a person is able to accurately state whether the colorful stimuli should be named 'red' or 'green', then the content of a color representation seems to determine that the represented color belongs to some category $C$ which fits one of the linguistic descriptions. A purely relational representation is unlikely to support such classification, as it simply represents that a color $X$ stands is some relations to other colors without containing any information about the color category of $X$. Of course, classification abilities in blindsight are severely limited, as color recognition is not successful outside the forced-choice paradigm, i.e. in situations in which there is no closed set of options. However, despite these differences in performance, I believe that results concerning blindsight provide support for a qualitative thesis that individuals with blindsight represent color not only relationally but also categorically.

However, there are data suggesting that color representations in blindsight do not represent surface colors but merely reflected colors. ${ }^{2}$ A crucial experiment conducted by Kentridge and colleagues (Kentridge et al. 2007) tested the color contrast perception abilities of a blindsighted individual, D.B. In order to achieve color constancy and represent surface colors, a visual system must distinguish variations in wavelength distribution arising from differences in luminance from those arising from reflectance properties of surfaces. An important heuristic used in achieving this goal consist in processing color contrast by comparing the light reflected from neighboring surface, as the relative differences are likely to remain the same despite changes in luminance. While color contrast perception is an important mechanism for achieving color constancy, in some circumstance it can lead to illusory perception. In particular, when two objects with the same light-reflectance properties are presented on a colorful, graduated background, such that each object is surrounded by a background of different color, they are inaccurately experienced as having distinct colors. In Kentridge et al.'s study, patient D.B. participated in a forced-choice task in which he was presented with a stimulus composed of a pair of discs with the same reflectance properties positioned on a (a) uniform colorful background, or (b) graduated colorful background. The two

\footnotetext{
${ }^{2}$ According to Phillips (2018), relying on theory of perception proposed by Burge (2010), such data suggest that what happen in blindsight is not in fact perception. I do not aim to evaluate this claim as my goal is only to show that even if blindsight is a form of unconscious perception, the studies on blindsight do not provide a justification for the equivalence thesis concerning color content.
} 
variants of the stimulus were presented one after another and the task was to indicate in which case the pair of discs better matched each other. A normally sighted person would perceive the disks on the uniform background as more similar. This was the result when stimuli were presented in a part of D.B.'s visual field unaffected by blindsight. However, when presented in the affected part of the visual field, discs in both variants of the stimulus appeared equally similar to D.B. This strongly suggests that unconscious color perception in blindsight lacks the crucial constancy mechanism related to contrast perception, and so it is likely that surface colors are not unconsciously represented in blindsight. Such results show that empirical investigations concerning blindsight do not provide support for the equivalence thesis.

However, because there are also pre-cortical mechanisms contributing to color constancy (see Spitzer and Rosenbluth 2002 for a detailed model concerning the role of retinas and lateral geniculate nucleus), one may propose that despite blindsight some form of surface colors perception is preserved. The crucial question is whether such precortical constancy mechanisms are sufficient for surface colors perception in a situation, occurring in blindsight, when important cortical structures, which usually further process input from pre-cortical mechanisms, do not function. In particular, it may be the case that even if some information regarding constancy is computed pre-cortically, without cortical mechanisms it cannot be used to form a proper representation of surface colors.

While I do not believe that the current empirical state of the art allows a definitive answer to this issue, Kentridge et al. (2007, pp. 15130-15131) provide an important argument which suggests that color constancy, and so surface colors perception, does not occur when relevant mechanisms in primary visual cortex are not available. It is so because pre-cortical cells do not demonstrate chromatic spatial opponency, i.e., they cannot respond to differences in chromatic properties of neighboring surfaces. Because, as noted by Spitzer and Rosenbluth, human color constancy works only when a stimulus is not presented alone but is surrounded by other stimuli (Brill and West 1986; TiplitzBlackwell and Buchsbaum 1988), lack of pre-cortical ability to compare spatially distinct surfaces suggests that without cortical mechanisms surface color representation cannot be achieved. It should be noted that Spitzer and Rosenbluth (2002) observe that not only neighboring surfaces but also spatially distant surfaces may contribute to the color constancy of a stimulus and this distal contribution may arise due to pre-cortical mechanisms in the lateral geniculate nucleus. Nevertheless, if such pre-cortical influences were sufficient for achieving color constancy without availability of mechanisms in the primary cortex, then some constancy effects should be apparent in the Kentridge et al. (2007) study, because presenting stimuli on a graduated colored background, significantly larger from the stimulus itself, allows not only for processing chromatic properties of neighboring areas, but also those possessed by areas spatially separated from the target stimulus.

Furthermore, one may believe that people affected by blindsight may have quite elaborate abilities to process color information, as it has been reported that they experience conscious, colorful afterimages in the blind part of visual field (see Weiskrantz et al. 2002). Furthermore, conscious, colorful phosphenes can appear in blind field as a result of transcranial magnetic stimulation (Silvanto et al. 2007). However, the presence of such phenomena is unlikely to provide support for a thesis that unconscious color perception in blindsight represents surface colors. In particular, 
there are reasons to believe that the appearance of conscious afterimages and phosphenes in blind field is in fact partially caused by activity in the remaining healthy parts of the primary visual cortex (see Brogaard 2011 for similar reasoning). The colorful phosphenes can be generated by transcranial magnetic stimulation after color adaptation. For instance, when a uniform red color is presented for a time needed to induce adaptation, transcranial magnetic stimulation is likely to produce red phosphenes. However, as shown by Silvanto et al. (2008), in case of individuals with blindsight unilateral transcranial magnetic stimulation of the damaged hemisphere do not produce colorful phosphenes in the blind field despite adaptation. Such colorful phosphenes occur when stimulation is bilateral, but then their color is determined by the stimulus to which the healthy part of visual cortex was adapted. For instance, when a red color is presented in blind half of visual field and a green color in normal half, then bilateral stimulation produces green, but not red, phosphenes throughout the whole visual field. In contrast, when both red and green stimuli are presented to healthy subjects, bilateral stimulation causes perception of both red and green phosphenes.

Furthermore, even if colorful afterimages and phosphenes could be genuinely produced in the blind field without a significant contribution from healthy parts of primary visual cortex, their mere presence would not provide a justification for unconscious perception of surface colors. To provide such evidence would require that the qualities of afterimages and phosphenes are such that they reflect rules of color constancy. In fact, studies on healthy participants show that color of an afterimage depends on context in which the initial stimulus was presented, and so is in an important respect determined by mechanisms of color contrast perception (see Zeki et al. 2017). However, such results have not been obtained in studies of individuals with blindsight, and are unlikely to be obtained given that Kentridge et al. (2007) showed that color contrast perception is not preserved in blindsight. Overall, empirical results concerning blindsight do not provide support for a thesis that conscious and unconscious color representations have the same type of content. While it may be maintained that colors in blindsight are represented categorically it is doubtful that surface colors are represented.

\section{Priming Studies and Categorical Perception}

Empirical evidence in favor of unconscious perception is not restricted to studies investigating dysfunctions of perceptual mechanisms like blindsight. In the context of color perception, the most important experimental paradigm used to illustrate the unconscious processing of color information in a properly functioning visual system is metacontrast masking (e.g., Norman et al. 2014; Ro et al. 2009; Schmidt 2002). In a standard metacontrast masking experiment, participants are presented with two successive colorful stimuli positioned in the same place: a circle and an annulus. A rapid presentation of an annulus after a brief presentation of a circle ensures the circle is not consciously perceived. In consequence, while two colorful stimuli are presented, a participant is only aware of the annulus. The usual task it to recognize, by choosing one of two options, the color of the annulus. It has been observed that the reaction time needed to provide the answer is shorter if the color of the annulus is the same as the color of the masked circle. This suggests that the color of the circle is processed unconsciously and facilitates the processing of an annulus of the same color. Unlike 
the case of blindsight, unconscious color perception occurs not in virtue of a disturbance in the function of the visual cortex, but due to a specific sequence and presentation time of stimuli.

While empirical investigations suggest that unconscious perception in blindsight is only able to represent reflected color and not surface color, there are results concerning metacontrast masking that show genuine surface color perception. Tests have shown the priming effect of unconsciously perceived circular stimuli on consciously perceived annulus occurs when both stimuli are presented under different illuminances despite possessing the same surface color (Norman et al. 2014). In consequence, while the presented surface properties were the same, the parameters of reflected light were different. The results show that the priming effect is preserved despite changes in luminance. In consequence, it seems that despite lack of conscious color awareness, constancy mechanisms operate successfully, and unconsciously perceived colorful stimuli are represented in terms of surface color.

It should be noted that the conclusion detailed above is not completely without controversy. For instance, Breitmeyer et al. (2004) observed that in a metacontrast masking task a white priming stimulus produced results more similar to those obtained when using a green priming stimulus than those obtained using a blue priming stimulus. The authors proposed that this effect arose because of features of the display screen, due to which green-related wavelengths and blue-related wavelengths did not equally contribute to the white stimulus, but instead the white stimulus was composed mostly of green-related wavelengths. Such an observation may suggest that performance during a metacontrast task is not determined by representational content concerning surface color properties but by representations with some lower-level content regarding merely wavelength combinations. Nevertheless, the evidence in favor of surface property perception obtained in metacontrast studies is stronger than that of blindsight. In consequence, I believe that it can be rationally maintained that such studies provide support for a thesis that there is a form of unconscious perception in which properties of colorful stimuli are represented as surface colors.

However, the situation differs when a distinction between relational and categorical color representations is considered; the results obtained in metacontrast studies do not provide support for a thesis that colors are unconsciously represented in a categorical way. The results obtained can be explained by referring only to the information provided by relational colors representations. As stated earlier, in metacontrast studies two stimuli are presented: a consciously perceived annulus and unconsciously perceived circular prime. The priming effect occurs when color of a prime is sufficiently similar to the color of annulus. The annulus is perceived consciously, and so it may be assumed that its color is represented both relationally and categorically. Nevertheless, there is no reason to postulate that the unconscious priming stimulus is represented categorically. The relational representation can convey information that the priming color stand in a certain relation of similarity, or even that it is identical, to the annulus color. If the represented relation indicates a high level of similarity, the priming effect occurs. On the other hand, if the similarity is low, this effect is not present. In consequence, to explain differences between situations in which priming occurs from those in which it is absent, there is no need to postulate that the priming color is also categorically represented, for instance as 'red' or 'green'. It is sufficient that it is represented as some color standing in a certain relation to the annulus color. 
An analogous line of reasoning applies to priming studies concerning phenomenon of visual neglect. Visual neglect is a condition typically resulting from damage in the right middle cerebral artery territory that often causes people to be unaware of stimuli occurring in the contralesional side of space (Cohen et al. 1995; Phillips 2016). A visual search study explicitly concerning unconscious color perception in individuals with visual neglect has been conducted by Kristjánsson et al. (2009). In visual search studies, the task of participants is to find a target possessing specific features despite the presence of distractors. One of the phenomena related to visual search is repetition priming: if the target has the same features in subsequent trials - for instance the task is to find a red target - the search is more efficient during the second trial. Kristjánsson et al. (2009) observed that when a target is presented in the neglected space, participants often fail to be aware of its presence. However, repetition priming is observed when the task during a subsequent trial is to find a target of the same color as in the first trial, this time positioned outside the neglected space. Such a result suggests that despite lack of awareness caused by visual neglect, color information is unconsciously processed. However, as in the case of metacontrast masking, such a result can be explained in terms of merely relational color representation. The priming effect may arise from the fact that the color of a consciously perceived target on a subsequent trial is represented as highly similar to the neglected color from the previous trial. In consequence, while the consciously perceived color is represented both categorically and relationally, in order to account for repetition priming it is not necessary to postulate that also neglected color was represented in a categorical way. Because of that the results of Kristjánsson et al. (2009) are consistent both with categorical and merely relational interpretation of unconscious colors.

The above considerations show that priming studies do not provide support for the equivalence thesis concerning unconscious color perception. The results of the studies considered do not allow differentiating whether conscious and unconscious color representations have distinct or equivalent types of content, as these results can be explained by referring to relational, and not categorical, unconscious color representations.

\section{Dichoptic Color Fusion and Unconscious Color Mixing}

In investigations concerning unconscious perception, several paradigms are used in which conscious processing is blocked by presenting two distinct stimuli simultaneously or in rapid succession (see Fogelson et al. 2014 for a review). For instance, in the continuous flash suppression paradigm, a conscious perception of a stimulus presented to one eye is masked by a dynamically changing pattern presented to the other eye (e.g., Tsuchiya and Koch 2005). Similarly, in a paradigm known as chromatic flicker fusion, two oppositely colored figures positioned on a uniform background are successively presented to both eyes (e.g., Hoshiyama et al. 2006). As a result, only a uniform background is perceived, and the figures are not consciously accessible. The most important of such paradigms in the study of unconscious color perception is dichoptic color fusion. In this case, two stimuli with opposite color contrast are simultaneously presented such that each is perceived by only one eye (Block 2015; Moutoussis and Zeki 2002). For example, one stimulus may be a green figure on a red background and a second a red figure on a green background. In consequence of this presentation, a 
person consciously perceives neither of the presented colors but instead a uniformly colored image. When green and red stimuli are used, the consciously seen image is yellow, consistent with the principles of additive color mixing. This result suggests that the colors of stimuli are processed unconsciously in such a way that information regarding colors is combined, leading to a conscious perception of a novel color.

However, similar to metacontrast masking studies, such results do not provide support for a thesis that unconsciously processed colors are represented categorically. When dichoptic fusion occurs, three colors are perceived. At first, two of them, red and green, are perceived unconsciously, and a third, yellow color is subsequently perceived consciously as a result of mixing of green and red. Because the yellow color is perceived consciously, it is likely that it is represented both relationally and categorically. On the other hand, there is no justification for postulating that the unconsciously perceived green and red are also represented categorically; the results of dichoptic color fusion can be explained by referring merely to relational color representations. According to this option, two unconsciously perceived colors are represented as colors $X$ and $Y$ which stand in some relations determining their position in a color space. When fusion occurs, the relational representations of $X$ and $Y$ are combined in accordance with the rules of additive color mixing, and a new representation is created, representing a color $Z$, possessing a new pattern of relations. Finally, the color $Z$ reaches a conscious level of processing and as a result is also represented categorically as yellow. In consequence, explaining dichoptic color fusion does not require postulating unconscious, categorical color representations.

The considerations outlined above show that the major examples of empirical investigations that aim to demonstrate unconscious color perception do not provide support for the equivalence thesis. In blindsight, it is likely that only reflectance and not surface colors are represented, and the results of priming studies as well as dichoptic color fusion results can be explained without postulating unconscious categorical color perception. Nevertheless, one may claim that while such studies do not separately support the equivalence thesis, such support is provided by their conjunction. In particular, if the success of blindsighted individuals in forced-choice tasks suggests the presence of categorical unconscious color perception and there are results suggesting surface color perception in metacontrast tasks, then it may seem that unconscious color perception can categorically represent surface colors. Nevertheless, such reasoning is problematic, as unconscious perception in not a homogenous phenomenon. While the number of extant types of unconscious perception is controversial (see Block 2015; Breitmayer 2015), it is plausible that the unconscious perception occurring in blindsight is of a different type than the unconscious perception occurring in metacontrast studies. In the case of blindsight, conscious processing is blocked due to a lack of normal activity of the primary visual context despite the fact that colorful stimuli are presented in a way that is not different from typical cases of conscious color perception. The opposite is true in metacontrast masking, where the mechanisms of visual perception are fully functional, but the conscious processing is blocked by the specific way in which stimuli are presented. Such a situation does not justify that there is a form of unconscious color perception which categorically represents surface colors. It still may be the case that there is one form of unconscious color perception, obtained due to unusual stimuli presentation, in which properties of colorful stimuli are not represented categorically but are represented as surface colors. Furthermore, a distinct form of unconscious color perception may occur, obtained due to certain disturbances 
in primary visual cortex functioning, in which properties of colorful stimuli are represented categorically but not as surface colors.

Relying on the above observations, one may ask whether there is some alternative way in which a justification for the equivalence thesis regarding color perception can be obtained. A possible idea is to refer to electrophysiological experiments concerning categorical color perception (see Section 1). In particular, it has been observed that when participants are presented with a change detection task with a change that replaces a color with a color from the same or a different category, greater visual mismatch negativity is recorded when a different-category change occurs. Visual mismatch negativity is a component of the event-related potential indicating pre-attentive visual change detection. Because of the pre-attentive nature of detection, some authors believe that results concerning visual mismatch negativity suggest unconscious categorical color processing (see Thierry et al. 2009). However, in the electrophysiological studies considered, no paradigm that seeks to block conscious processing, such as metacontrast masking, was applied. Thus, it is not completely clear how the colorful stimuli were processed and what causes categorical effects to arise. Furthermore, it cannot be simply assumed that preattentive processing entails unconscious processing, as the link between attention and consciousness remains a matter of ongoing debate (see Jennings 2015; Mole 2008; Prinz 2012 for overviews of various positions).

\section{Conclusions}

The thesis that conscious and unconscious representations have the same type of content is postulated in the context of influential theories of consciousness. Furthermore, it is not uncommon to claim that such an equivalence thesis is justified by empirical investigations concerning unconscious vision. Nevertheless, the most important empirical studies regarding unconscious color perception concerning blindsight, metacontrast masking, neglect studies, and dichoptic color fusion, do not, in fact, provide support for this claim. In the case of blindsight, surface colors are unlikely to be represented, and the results of priming studies and dichoptic color fusion experiments can be explained without postulating categorical unconscious color representations.

Acknowledgements The author would like to thank the anonymous reviewers and the journal editor for comments concerning the paper. Special thanks for Paweł Zięba who inspired me to address the topic of unconscious color perception.

Funding The work was supported by the National Science Center (Poland) grant 2018/31/D/HS1/00363.

Declarations I comply with the Ethical Standards of "Review of Philosophy and Psychology".

Conflict of Interest The author declares that he has no conflict of interest.

Informed Consent and Animal Welfare The research did not involve human participants or animals.

Open Access This article is licensed under a Creative Commons Attribution 4.0 International License, which permits use, sharing, adaptation, distribution and reproduction in any medium or format, as long as you give 
appropriate credit to the original author(s) and the source, provide a link to the Creative Commons licence, and indicate if changes were made. The images or other third party material in this article are included in the article's Creative Commons licence, unless indicated otherwise in a credit line to the material. If material is not included in the article's Creative Commons licence and your intended use is not permitted by statutory regulation or exceeds the permitted use, you will need to obtain permission directly from the copyright holder. To view a copy of this licence, visit http://creativecommons.org/licenses/by/4.0/.

\section{References}

Anaya, A., and S. Clarke. 2017. Naïve realism and unconscious perception: A reply to Berger and Nanay. Analysis 77 (2): 267-273.

Berger, J., and B. Nanay. 2016. Relationalism and unconscious perception. Analysis 4: 426-433.

Block, N. 2015. The Anna Karenina principle and skepticism about unconscious perception. Philosophy and Phenomenological Research 93 (2): 452-459.

Boyer, J.L., S. Harrison, and T. Ro. 2005. Unconscious processing of orientation and color without primary visual cortex. PNAS 102 (46): 16875-16879.

Breitmeyer, B.G. 2015. Psychophysical "blinding" methods reveal a functional hierarchy of unconscious visual processing. Consciousness and Cognition 35: 234-250.

Breitmeyer, B.G., T. Ro, and N.S. Singhal. 2004. Unconscious color priming occurs at stimulus- not perceptdependent levels of processing. Psychological Science 15 (3): 198-202.

Brent, P.J., C. Kennard, and K.H. Ruddock. 1994. Residual colour vision in a human hemianope: Spectral responses and colour discrimination. Proceedings of the Royal Society 256: 219-225.

Brill, M.H., and G.W. West. 1986. Chromatic adaptation and color constancy: A possible dichotomy. Color Research Applications 11: 196-204.

Brogaard, B. 2011. Color experience in blindsight? Philosophical Psychology 24 (6): 767-786.

Burge, T. 2010. The origins of objectivity. Oxford: Oxford University Press.

Clifford, A., A. Holmes, I.R.L. Daviesa, and A. Franklin. 2010. Color categories affect pre-attentive color perception. Biological Psychology 85: 275-282.

Clifford, A., A. Franklin, A. Holmes, V.G. Drivonikou, E. Ozgen, and I.R.L. Davies. 2012. Neural correlates of acquired category effects. Brain and Cognition 80: 126-143.

Cohen, A., R.B. Ivry, R.D. Rafal, and C. Kohn. 1995. Activating response codes by stimuli in the neglected visual field. Neuropsychology 9 (2): 165-173.

Cowey, A. 2004. Fact, artefact, and myth about blindsight. The Quarterly Journal of Experimental Psychology Section A: Human Experimental Psychology 57 (4): 577-609.

Davidoff, J., and J. Fagot. 2010. Cross-species assessment of the linguistic origins of color categories. Comparative Cognition and Behavior Reviews 5: 100-116.

Dretske, F. 2006. Perception without awareness. In Perceptual experience, ed. T.S. Gendler and J. Hawthorne, 147-180. Oxford: Oxford University Press.

Fogelson, S.V., P.J. Kohler, K.J. Miller, R. Granger, and P.U. Tse. 2014. Unconscious neural processing differs with method used to render stimuli invisible. Frontiers in Psychology 5: 601. https://doi.org/10. 3389/fpsyg.2014.00601.

Franklin, A., A. Clifford, E. Williamson, and I. Davies. 2005a. Color term knowledge does not affect categorical perception of color in toddlers. Journal of Experimental Child Psychology 90: 114-141.

Franklin, A., and I.R.L. Davies. 2004. New evidence for infant colour categories. British Journal of Developmental Psychology 22: 349-377.

Franklin, A., M. Pilling, and Davies. 2005b. The nature of infant color categorization: Evidence from eye movements on a target detection task. Journal of Experimental Child Psychology 91: 227-248.

Franklin, A., O. Wright, and I.R.L. Davies. 2009. What can we learn from toddlers about categorical perception of color? Comments on Goldstein, Davidoff, and Roberson. Journal of Experimental Child Psychology 102: 239-245.

Hoshiyama, M., R. Kakigi, Y. Takeshima, K. Miki, and S. Watanabe. 2006. Priority of face perception during subliminal stimulation using a new color-opponent flicker stimulation. Neuroscience Letters 402: 57-61.

Jennings, C.D. 2015. Consciousness without attention. Journal of the American Philosophical Association 1 (2): $276-295$. 
Kay, P., and T. Regier. 2006. Language, thought and color: Recent developments. Trends in Cognitive Sciences 10 (2): 51-54.

Kentridge, R.W. 2015. What is it like to have type-2 blindsight? Drawing inferences from residual function in type-1 blindsight. Consciousness and Cognition 32: 41-44.

Kentridge, R.W., C.A. Heywood, and L. Weiskrantz. 2007. Color contrast processing in human striate cortex. PNAS 104 (38): 15129-15131.

Kristjánsson, A., P. Vuilleumier, P. Malhotra, M. Husain, and J. Driver. 2009. Priming of color and position during visual search in unilateral spatial neglect. Journal of Cognitive Neuroscience 17 (6): 859-873.

Mole, C. 2008. Attention and consciousness. Journal of Consciousness Studies 15 (4): 86-104.

Moutoussis, K., and S. Zeki. 2002. The relationship between cortical activation and perception investigated with invisible stimuli. PNAS 99 (14): 9527-9532.

Norman, L.J., K. Akins, C.A. Heywood, and R.W. Kentridge. 2014. Color constancy for an unseen surface. Current Biology 24: 2822-2826.

Ocelák, R. 2016. "Categorical perception" and linguistic categorization of color. Review of Philosophy and Psychology 7: 55-70.

Palmer, S. 1999. Vision science: Photons to phenomenology. Cambridge, MA: MIT Press.

Peebles, G. 2017. Reflexive theories of consciousness and unconscious perception. Philosophical Psychology 31 (1): 25-43.

Phillips, I. 2016. Consciousness and criterion: On Block's case for unconscious seeing. Philosophy and Phenomenological Research 93 (2): 419-451.

Phillips, I. 2018. Unconscious perception reconsidered. Analytic Philosophy 59 (4): 471-514.

Phillips, I. 2020. Object files and unconscious perception: A reply to Quilty-Dunn. Anaysis 80 (2): $293-301$.

Prinz, J.J. 2012. The conscious brain: How attention engenders experience. New York: Oxford University Press.

Quilty-Dunn, J. 2019. Unconscious perception and phenomenal coherence. Analysis 79 (3): 461-469.

Ramsøy, T.Z., and M. Overgaard. 2004. Introspection and subliminal perception. Phenomenology and the Cognitive Sciences 3: 1-23.

Reingold, E.M. 2004. Unconscious perception: Assumptions and interpretive difficulties. Consciousness and Cognition 13: 117-122.

Ro, T., N.S. Singhal, B.G. Breitmeyer, and J.O. Garcia. 2009. Unconscious processing of colorand form in metacontrast masking. Attention, Perception, \& Psychophysics 71 (1): 95-103.

Rosenthal, D.M. 2005. Consciousness and mind. Oxford: Oxford University Press.

Rosenthal, D.M. 2010. How to think about mental qualities? Philosophical Issues 20: 368-393.

Schlicht, T. 2012. Phenomenal consciousness, attention and accessibility. Phenomenology and the Cognitive Sciences 11: 309-334.

Schmidt, T. 2002. The finger in flight: Real-time motor control by visually masked color stimuli. Psychological Science 13 (2): 112-118.

Silvanto, J., A. Cowey, N. Lavie, and V. Walsh. 2007. Making the blindsighted see. Neuropsychologia 45: 3346-3350.

Silvanto, J., A. Cowey, and V. Walsh. 2008. Inducing conscious perception of colour in blindsight. Current Biology 18 (20): R950-R951.

Snodgrass, M. 2004. The dissociation paradigm and its discontents: How can unconscious perception or memory be inferred? Consciousness and Cognition 13: 107-116.

Spitzer, H., and A. Rosenbluth. 2002. Color constancy: The role of low-level mechanisms. Spatial Vision 15 (3): 277-302.

Stoerig, P., and A. Cowey. 1992. Wavelength discrimination in blindsight. Brain 115: 425-444.

Taylor, H. 2019. Fuzziness in the mind: Can perception be unconscious? Philosophy and Phenomenological Research 101: 383-398. https://doi.org/10.1111/phpr.12592.

Thierry, G., P. Athanasopoulos, A. Wiggett, B. Dering, and J.-B. Kuipers. 2009. Unconscious effects of language-specific terminology on preattentive color perception. PNAS 106 (11): 4567-4570.

Tiplitz-Blackwell, K., and G. Buchsbaum. 1988. Spatial and chromatic parameters of color contrast. Color Research and Application 13: 166-173.

Tsuchiya, N., and C. Koch. 2005. Continuous flash suppression reduces negative afterimages. Nature Neuroscience 8: 1096-1101.

Tye, M. 1995. Ten problems of consciousness: A representational theory of the phenomenal mind. Cambridge, MA: MIT Press.

Vosgerau, G., T. Schlicht, and A. Newen. 2008. Orthogonality of phenomenality and content. American Philosophical Quarterly 45: 329-348.

Weiskrantz, L. 2009. Is blindsight just degraded normal vision? Experimental Brain Research 192: 413-416. 
Weiskrantz, L., A. Cowey, and I. Hodinott-Hill. 2002. Prime-sight in a blindsight subject. Nature Neuroscience 5 (2): 101-102.

Zeki, S., S. Cheadle, J. Pepper, and D. Mylonas. 2017. The constancy of colored after-images. Frontiers in Human Neuroscience 11: 229.

Zięba, P.J. 2019. Naïve realism about unconscious perception. Synthese 196: 2045-2073.

Publisher's Note Springer Nature remains neutral with regard to jurisdictional claims in published maps and institutional affiliations. 